\title{
Fundamentos epistemológicos en la investigación científica
}

\section{Resumen}

Como consecuencia del pragmatismo impuesto por el positivismo, se ha empobrecido la formación humanística de los egresados de las instituciones educativas en general, lo que ha ocasionado la distorsión del verdadero objeto de estudio de la ciencia, contemplando sólo lo mensurable y dejando de lado la expresión y sentimientos humanos que definen al sujeto. La cotidiana labor docente requiere conocer los fundamentos epistemológicos, teóricos y prácticos de la Psicología, conocer los procesos de aprendizaje facilitará la enseñanza. nos proponemos dar respuesta a una nutrida y variada lista de paradojas que la Psicología tradicional, ha ido engrosando nuestras agendas académicas y que hemos venido postergando durante varias décadas, deuda que los psicólogos hemos contraído con la educación, con la comunidad académica y con la sociedad.

\section{Palabras clave:}

Positivismo, humanismo, paradoja, epistemología.

\section{Epistemological basis in scientific research}

\begin{abstract}
As a result of pragmatism imposed by positivism, graduate humanistic education from institutions at large has been impoverished. This situation has produced the distortion of the actual object of study of science, regarding only the measurable and leaving aside the expression and emotions that define the human being. Everyday teaching requires knowing the epistemological, theoretical, and practical foundations of Psychology, since being aware of the learning process will facilitate education. We intend to respond to a large and varied list of paradoxes with which traditional Psychology has filled our academic agendas, and we have been putting off for decades. This is a debt that psychologists have incurred to education, the academic community, and society.
\end{abstract}

\section{Keywords:}

Positivism, humanism, paradox, epistemology. 


\section{Introducción}

Frente a este panorama, se propone dar inicio a la preocupación, de dar a nuestra mirada un sentido epistemológico, que permita nutrirnos de un acercamiento a la ciencia.

En efecto, muchas teorías psicológicas hasta el momento se han dedicado únicamente en fragmentar el comportamiento humano, privilegiando algunos aspectos e ignorando muchos otros, estamos convencidos que podemos intentar reparar tal fragmentación e intentar explicarnos mucho mejor el comportamiento humano del que disponíamos hasta hace unas décadas.

Las deficiencias que se señalan, ya habían sido advertidas hace algunas décadas atrás por José Antonio Encinas (1934), y Cesar Augusto Guardia Mayorga, quienes propusieron no solo la necesidad de construir una Psicología del Hombre Concreto, es decir, afincado en un contexto histórico, social, cultural, pues no existe ningún hombre en abstracto, autista, fuera de cualquier tipo de contexto. Esta teoría debimos haberla construido mucho antes, sin embargo, esta tarea se fue postergando indefinidamente. El VI Congreso Internacional de Educación, nos permite la posibilidad de iniciar esta discusión, como una modesta contribución de la Academia Peruana de Psicología a la educación Peruana.

Encinas expone con mucha anticipación que el estudio del hombre debe hacerse necesariamente con arreglo a sus condiciones de vida, respetando la singularidad de su subjetividad, su inserción al contexto social donde vive y su comportamiento expresará la dinámica de esta relación con el medio no solo geográfico natural, también las condiciones específicas de su existencia individual.

Una de las deficiencias más notorias dentro de la investigación científica, sobre todo en el campo educativo se encuentra relacionada con la ausencia de una teoría confiable en psicología cuyo sustento epistemológico le permita orientar la investigación en mejores condiciones, aquí se advierten una serie de paradojas que no han sido resueltas adecuadamente.

Esta es una lista de paradojas podemos enumerarlas brevemente y que nos proponemos abordarlas, la paradoja se expresa cuando creemos disponer de la información necesaria para explicar y nos percatamos que estas no son satisfactorias, mucho menos convincentes:

1.- Explicar mejor la singularidad del comportamiento humano, (Encinas 1934, Galperin, 1976)

2.- Esto servirá para entender que tanto los miembros de las poblaciones, como las poblaciones mismas son necesariamente heterogéneos (Martel 2015).

3.- Esto implica abordar de otro modo la comprensión de las investigaciones tradicionales que privilegiaban las pocas generalizaciones, ignorando las singularidades (Stake, 199).

4.- Las pocas y gruesas generalizaciones que se obtenían en las investigaciones tradicionales no lograron resolver los problemas colectivos prácticos, de donde provienen. Tampoco conseguían resolver los problemas teóricos individuales de los investigadores. Esto explica en gran medida una de las deficiencias más notorias de la actividad académica en nuestra comunidad (Sandoval 2002).

5.- Esto nos permitirá entender el origen de los problemas en el aprendizaje, tan comunes en nuestra labor cotidiana, problemas que casi nunca se resuelven satisfactoriamente. En muy pocas ocasiones encontramos personas que se aproximen a los modelos paradigmáticos que hemos construido (Martel 2006). 
6.- El dimorfismo sexual cerebral, el machismo, sexualidad, género y la disfuncionalidad familiar imponen improvisaciones en la crianza de los niños que afecta severamente su desarrollo posterior (Denegri 2015)

7.- Comprenderemos mejor la disfuncionalidad familiar, asociada a la mayor parte de los problemas en el aprendizaje (Martel 2006).

8.- Comprender mejor la influencia de las supersticiones y la cultura en el comportamiento de las personas, dentro y fuera de la actividad académica (Martel 2014).

9.- Comprender mejor el "componente espiritual", como una construcción subjetiva nuestra y no ajena o externa. Construcción que se elabora a lo largo de nuestra vida orientada hacia el bienestar individual y colectivo (Rand 2008, Martel 2014, Savater 2005).

10.- Podemos acceder a la construcción de una teoría que pueda superar las limitaciones de las anteriores ya que actualmente estamos lejos de contar con una teoría unificada de la psicología, esto no impide que construyamos una orientada en esta dirección (MartelUrbano 2011).

11.- Criticar teorías defectuosas, podemos reconocerlas como ignorancias disfrazadas, corregir las deficiencias y omisiones o sustituir teorías (Savater 2005, Popper 1974, Mosterín 2006).

12.- Esto nos permitirá aproximarnos a criterios de normalidad más confiables que el criterio estadístico y que este no es el único criterio disponible y menos aún el más confiable (Martel-Urbano, 2011).

13.- Una última paradoja, los problemas anteriores, los hemos construido con nuestra ignorancia (Popper 1974) a los cuales se han añadido otros fomentados por el consumismo enajenante (Beigbeder 2002).

\section{Fundamentación de las propuestas}

\section{Sobre la singularidad del comportamiento humano.}

Sobre la singularidad del comportamiento humano, existen aproximaciones teóricas confiables, a partir de la naturaleza multiparamétrica de la Actividad Psíquica (Anojín 1984, Maturana 1996), según este último, se requiere corregir la creencia que todo estímulo genera necesariamente una respuesta, lo que provoca un estímulo es una serie de cambios de estado en el sistema nervioso, confirmándose su naturaleza multiparamétrica. Según sea la intensidad de dichos cambios, las actividades más intensas logran imponerse sobre las menos intensas que son muchas y que pasan completamente desapercibidas.

Es a partir de aquellas intensas, que empieza a elaborarse una compleja actividad de procesamiento e interpretación subjetiva que puede expresarse en una respuesta orientada en alguna dirección. Si es que el procesamiento es adecuado y se identifica el estímulo, la respuesta se orienta adecuadamente, esto supone la preexistencia de los saberes previos (Ausubel 1982) o más bien según sea el ordenamiento jerárquico de nuestra actividad psíquica (Galperin 1976).

Dicho ordenamiento, es singular único e irrepetible en cada uno de nosotros, y con arreglo al contexto en el que nos encontremos y las contingencias específicas de la experiencia. Esto se explica mucho más de acuerdo a nuestras diferencias individuales de sexo, edad, cultura, economía, creencias y supersticiones, expectativas y frustraciones, etc. 
El propio desarrollo de la sociedad genera modificaciones muchas de ellas irreversibles de acuerdo a las condiciones de vida de las personas, en nuestra experiencia cotidiana hemos asistido a los cambios más recientes como aquellas generadas por el impacto de la tecnología, la globalización de la economía, la corrupción generalizada que ha afectado a muchas instituciones, la inseguridad ciudadana, etc.

Estas nociones resultan imprescindibles si es que deseamos una formación humanística decorosa en nuestros estudiantes, para eso los maestros necesitamos de las herramientas intelectuales necesarias para construir un humanismo lejos de los dogmatismos y adoctrinamientos.

\section{Personas singulares, poblaciones heterogéneas}

En nuestra labor cotidiana de docentes nos percatamos que los estudiantes con quienes compartimos experiencias académicas, no constituyen grupos homogéneos, a pesar de compartir grupos con similares condiciones de edad, grado de instrucción, etc. A pesar de estos datos comunes, comprobamos que trabajamos con grupos heterogéneos, ya sea por razones estructurales o atendiendo a las diferencias individuales de sus miembros.

Por lo tanto el tratamiento de esta dinámica debe hacerse atendiendo a las diferencias señaladas, esto requiere del docente una mayor capacidad de tolerancia, pues la falta de disposición para reconocer estas diferencias generan o agravan los problemas en el aprendizaje que comportan no solo los estudiantes, advertimos también en los adultos con quienes viven, aquí es necesario estar conscientes también de las dispedagogías en las que incurrimos los docentes a pesar del esfuerzo por evitarlas.

El desconocimiento de los fundamentos de la psicología hace que ignoremos la naturaleza humana y cometamos errores no solo conceptuales, a estos se añaden decisiones equivocadas y omisiones que afectan nuestra tarea cotidiana. Muchos maestros, han reparado ya estas deficiencias inducidas por teorías defectuosas de la psicología, sobre todo aquellas teorías que provienen de la etapa pre-científica de la psicología y que se mantienen por supersticiones ideológicas. Errores que pretendemos revertirlos a partir de encuentros y discusiones como en el presente congreso en el que participamos. En esto radica la inmensa importancia de actividades académicas.

En nuestro país, existen diversas culturas que se confrontan en cada experiencia migratoria, la migración interna se produce permanentemente y continuará corriendo por el centralismo, genera conflictos derivados de la confrontación entre culturas. Aquí se advierte no solo el desarraigo de los migrantes, se añade también la marginación en la que se encuentran las poblaciones rurales, tanto alto andinas como las selváticas. Revertir esta tendencia va llevar mucho más tiempo que la sustitución de los esquemas mentales de la población migrante.

\section{Nuevos enfoques en la investigación científica.}

Las ideologías de orientación positivista adolecen de una severa deficiencia: Desconocen la singularidad de comportamiento humano, el éxito logrado en las ciencias naturales y la tecnología se ha pretendido imponer al estudio del comportamiento humano sin éxito. Esto les ha llevado a privilegiar e imponer un modelo de investigación a la que se le ha venido a denominar como investigaciones cuantitativas, donde se sobrevalora el 
tratamiento de los datos estadísticos, ignorando a los individuos de quienes se obtienen dichos datos.

Esta tendencia ha llevado también a la metodolatría (Martel 2012), es decir la magnificación de los métodos en perjuicio de la fundamentación epistemológica de las teorías que sustentan la investigación. Esta tendencia tiene todavía mucha vigencia en la creencia que matematizando cualquier tipo de información, aun cuando esta sea errónea, las investigaciones adquieren de inmediato un "estatus científico". Cuando en realidad lo único que se reproduce es la caricatura que caracteriza a la indigencia académica.

Lo más sensato es en primer lugar, exigir una mayor consistencia en los supuestos teóricos que sustentan las investigaciones. En segundo lugar interpretar con amplitud los resultados, no solamente sesgando a los que aparentemente alcanza significación estadística y pueden ser objeto de generalizaciones, hay que interpretar también las singularidades. Muchas veces el desestimar tales singularidades ha llevado a desperdiciar información valiosa.

La falta de información para hacer interpretaciones con mayor profundidad de los resultados solo se explica por la indigencia epistemológica de las investigaciones, es necesario reparar a la brevedad esta deficiencia que nos expone a la caricatura que se expresa, muy brevemente: Investigaciones que no resuelven problemas prácticos en la sociedad, ni teóricos en los investigadores, generando un círculo vicioso que se repite, se requiere sustituirlo por otro círculo virtuoso que resuelva ambos problemas no solo prácticos también los teóricos.

Las limitaciones anteriores se advierten en los trabajos de investigación desarrollados para la obtención de los grados académicos en los egresados no solo en el pre grado en las distintas profesiones, esto es más lamentable en los profesionales que laboran en los servicios de salud y educación, donde se requiere con mayor urgencia claridad en su formación académico profesional que resuelva los problemas que confrontan los estudiantes y los pacientes usuarios de dichos servicios.

Se requieren nuevos enfoques que partan del reconocimiento de las severas limitaciones que nos imponen las investigaciones tradicionales impuestas por el positivismo, en la creencia que los problemas sociales y humanos pueden ser tratados del mismo modo cómo se abordan los problemas en las ciencias naturales.

Otra propuesta es darles un tratamiento más diversificado a la muestra agrupándolos por tercios: alto medio y bajo, Atendiendo al sexo: varones y mujeres. Puede ayudarnos a entender de alguna manera los puntajes obtenidos y explicar mejor los resultados, cuando se les incluye en un solo grupo, los puntajes se pierden y dejan de ser significativos.

\section{Muchos problemas les hemos creado por nuestra ignorancia}

En efecto, muchos de los problemas en el aprendizaje que confrontamos a diario han sido impuestos culturalmente, y derivan de supersticiones que la educación no ha podido superar y muchas veces se ha allanado a ellas, tal como ocurre con el consumismo, el machismo, por citar algunas de las supersticiones más comunes Esto no significan que sean las únicas, existen muchas otras que por ser cotidianas ya no llaman la atención, pero su presencia se advierte de inmediato.

Tal es el origen de muchos de los problemas en el aprendizaje que confrontamos y su falta de atención genera otros problemas mucho más complejos, como ocurre con el 
machismo, el cual ha experimentado diversas mutaciones a lo largo de la evolución de las sociedades. La discriminación que experimentan las mujeres, en algunas civilizaciones mucho más que en otras, ha afectado a toda la sociedad en su conjunto, cuya expresión se manifiesta actualmente en la disfuncionalidad familiar, la crianza de los hijos, etc.

El machismo orienta a hombres y mujeres en distintas direcciones, a los hombres les alienta a la autonomía, de ahí la tendencia a evadir responsabilidades, sobre todo en la renuencia a asumir la paternidad. En tanto que a la mujer le imponen la dependencia, la crianza de los hijos, la atención a las labores domésticas, etc. Estas tendencias se han reducido gradualmente en algunas capas de la sociedad, sin embargo, estamos muy lejos de haberla erradicado.

Los avances en reducir esta superstición son todavía insuficientes y continúan generando disfuncionalidad entre los miembros de las instituciones desde los hogares hasta los centros de enseñanza, instituciones laborales y otras formas de ocupación o empleo. Para reducir el impacto de esta forma de discriminación, se requiere que las instituciones educativas incorporen programas destinados a esta finalidad, se requiere también que las niñas tengan modelos femeninos deseables que se opongan a los modelos caricaturescos creados para el consumo desde los medios de comunicación.

El machismo deformante se advierte desde la crianza de los niños en los hogares, continúa en las escuelas, los medios de comunicación y otras actividades culturales. El consumismo impuesto desde los medios de comunicación ha acentuado la divergencia entre las lógicas masculina y femenina. Esta deficiencia se advierte en el trato que recibe el niño y la niña que no es igualitario sino discriminadora en cuanto a la identificación con el género al que pertenecen.

Entre hombres y mujeres existen diferencias, no solo hormonales, existen también diferencias en la constitución neuronal, fisiológica, funcional y psicológica. A estas diferencias se han añadido otras con fines comerciales otra mucha mayor: el consumismo compulsivo en la población, sobre todo en los sectores con menores recursos que derivan en el incremento de la falta de seguridad en la sociedad.

\section{Aproximaciones a la espiritualidad humana}

Algunas líneas arriba, se ha señalado que se advierte la naturaleza multiparamétrica de la Actividad Psíquica, esto supone que estamos en condiciones muy precarias para conocer la mayor parte de nuestra subjetividad que tiende al infinito por la inmensa cantidad de información que contiene y se mantiene ignorada, hasta que no alcance la intensidad requerida para estar medianamente conscientes de ellas (Rand 2008).

En el continuo de nuestra actividad psíquica hay una cantidad demasiado grande de representaciones ignoradas y se mantendrán en este estado mientras permanezcan en un estado de baja intensidad. Solo estamos conscientes de una parte demasiado pequeña que alcanza la intensidad requerida para estar conscientes de ellas, solo entonces generan un discreto. Toda esta compleja red de asociaciones se orientan al bienestar individual aunque muchas de estas resulten deficientes, y enajenantes.

Por lo tanto tiene sentido cuando se afirma que nuestros conocimientos serán necesariamente finitos, en tanto que nuestra ignorancia será infinita (Popper 1974) Esto explica ya cómo es que organizamos complejos modelos subjetivos sin habernos percatado de ellos y al no poder explicarlos, tratamos de racionalizarlos ingenuamente como una construcción espiritual, mística, esotéria, ajena a nosotros. 
De este modo es que surgen nuestras dependencias y nuestras fobias, nuestras esperanzas y frustraciones, según sean los modelos que hayamos construido durante nuestra experiencia personal. Estos modelos tienen desde luego un repertorio de cualidades, propiedades ideales a los que hemos convenido en llamarlas como valores, con arreglo a los cuales orientamos nuestro comportamiento.

De este modo también se han construido nuestras creencias ideológicas y la mayor parte de nuestras supersticiones, en lo que Rand (2008) define como las psicoepistemologías, Para tener una idea aproximada entre lo que es posible conocer acerca de nuestra subjetividad y lo que ignoramos de ella, comparemos la inmensa cantidad de información que procesamos de nuestra fisiología, del funcionamiento de nuestros órganos internos, casi toda esta variedad de información, permanece ignorada solo experimentamos bienestar cuando el funcionamiento es adecuado hasta que un malestar sin identificación nos da cuenta de alguna anomalía e intentamos atenderla mediante alguna acción voluntaria.

Y una vez restablecida la armonía, toda esta inmensa cantidad de información que fluye nuevamente pasa totalmente desapercibida y continuamos procesándolas sin ninguna interrupción, estemos conscientes o no de ellas. Más bien de lo que estamos conscientes es de una cantidad muy pequeña, en comparación a la información que pasa desapercibida.

Entender, cómo se construye la espiritualidad, nos conduce a la comprensión de cómo se construye la subjetividad humana (Martel y Urbano 2011) a partir de sucesivas aferentaciones en retorno multiparamétricas (Anojín 1987), este conocimiento no puede ser exclusividad de los psicólogos, debiera ser de conocimiento de cualquier persona que tenga la curiosidad intelectual para tal fin, debiera ser uno de los objetivos transversales de quienes tengan la necesidad de explicarse su propia naturaleza.

\section{Otras formas de enajenación}

Uno de los problemas que confronta actualmente nuestra sociedad es la inseguridad ciudadana y la corrupción de generalizada, detrás de estas formas de comportamiento antisociales, delincuenciales se encuentra el consumismo que alientan con fines comerciales (Beigbeder 2002), no solo los medios de comunicación, lo mismo ocurre con otros agentes, como las costumbres asociadas a celebraciones diversas donde nuevamente es el consumo lo que genera toda la dinámica.

Quienes muestran una mayor proclividad por el consumo son los niños, jóvenes y adolescentes, todos ellos con carencias afectivas (Beidbeder 2002) quien señala que los consumidores compulsivos no son las personas adultas que por la madurez cognitivo afectiva que han logrado alcanzan un buen acomodo al contexto social, sino son las personas que por su inseguridad son más susceptibles de incorporados como consumidores compulsivos.

En otros términos los consumidores compulsivos no son las personas exitosas, sino aquellos que por diversas razones son aquellas que padecen de un mal acomodo al contexto social en los que se encuentran. Los dichosos no consumen, los desdichados sí lo hacen y en exceso aumentando con esto su ya pesado infortunio. La educación tampoco ha previsto dentro de los programas curriculares algo que pueda orientar adecuadamente este tipo de comportamiento compulsivo.

Esto explica en gran medida no solo la corrupción en las instituciones, explica también la inseguridad en la que vivimos a diario, los cambios deben empezar por las instituciones 
educativas, sobre todo las superiores, estos cambios no se van a producir espontáneamente en la sociedad. Aunque reconocemos y alentamos los cambios individuales, estos seguirán siendo insuficientes para resolver los conflictos sociales que genera el consumismo compulsivo.

En el contexto que se describe, se requiere de un mayor liderazgo por parte de los docentes, sin embargo la falta de este, solo muestra, nuevamente precariedades en su formación profesional. Para que los docentes asuman el liderazgo paradigmático que deseamos es necesario dotarlos de las herramientas intelectuales que en la actualidad se dispones y una parte de ellas se encuentran contenidas en las propuestas que se han expuesto.

Con respecto al liderazgo educativo, nuevamente Encinas constituye en un paradigma digno a seguir y propone que para tal fin es necesario construir modelos paradigmáticos para orientar en esa dirección a estudiantes y profesores, educando para la ciudadanía y no con otros fines ajenos a ella.

\section{¿ Es posible controlar los medios de comunicación enajenantes?}

Colectivamente no, individualmente sí. La libertad de prensa no permite censurar a los medios, la autocensura es una caricatura, además las libertades individuales facultan a las personas a acceder sin restricción alguna incluso a contenidos aberrantes, enajenantes. Aquí nuevamente se requiere del liderazgo individual de maestros y padres a fin de orientar a los estudiantes a fin de encarar con una actitud crítica lo que los medios de comunicación difundan.

Lamentablemente la falta de liderazgo en los adultos lleva a los niños a estar expuestos durante largas horas a programas enajenantes de lo que se ha venido en denominar como la televisión basura a la que puede añadirse el internet y otros. Requerimos adquirir la competencia necesaria para orientarnos adecuadamente (Tobón 2010).

Discutir sobre los contenidos de los programas nos permite nuevamente a interpretar diversos puntos de vista donde el adulto con un mayor número de herramientas intelectuales a su disposición puede orientar y recomendar el acceso a determinados programas cuyos objetivos pueden advertirse de inmediato.

Mucho se comenta que los esfuerzos educativos de los maestros se colisionan con la competencia desleal de los medios, sin embargo es posible controlarlos a partir de la actitud crítica que el público adquiera y es de esperar que esta actitud crítica la obtuviesen en las instituciones educativas a las que el estudiante concurre a diario (Mosterín 2006)

No podemos esperar que los medios de comunicación se dediquen a la educación, ya que como empresas se dedican a la venta de productos, a fomentar el consumismo. Es a las instituciones educativas a las que les compete la noble tarea de educar a los estudiantes y a la formación de los ciudadanos. Abdicar a esta función que nos compete, equivale a admitir la marginalidad de las instituciones educativas, equivale a descalificarlas prematuramente. 


\section{Conclusiones y sugerencias}

Como consecuencia de estos objetivos que nos proponemos desarrollar, podemos precavernos de no continuar asumiendo decisiones erróneas en nuestra labor cotidiana en la docencia a partir del acceso a teorías más confiables en la enseñanza y el aprendizaje.

Se requiere asumir un mayor liderazgo académico a partir del fortalecimiento en la formación profesional de maestro, dotándolo de las herramientas intelectuales necesarias para tal fin. Este es el propósito que nos hemos impuesto, la presente propuesta es solo un fragmento que puede dar inicio o permitirá continuar con una tarea que ya ha comenzado en alguna otra experiencia académica anterior.

Es conveniente fortalecer la formación humanística de los egresados de las instituciones educativas a partir de las nociones mejor logradas por la psicología para explicar la complejidad del comportamiento humano.

Para esto se requiere sensibilizar a las autoridades educativas, quienes tomar decisiones para impulsar los cambios curriculares que se requieren con urgencia. Al mismo tiempo es necesario reconocer a quienes desempeñan acertadamente su labor docente, con ellos hemos adquirido una inmensa deuda que requiere honrarla a la brevedad y no esperar el reconocimiento póstumo.

Finlandia ha conseguido mejorar la calidad de la educación y constituye un claro ejemplo que es posible revertir estas complejidades logrando reducir la pobreza, una mejor calidad de vida, y una mayor seguridad para los miembros de la sociedad; a partir de la mejora en la calidad educativa.

La experiencia finlandesa nos muestra también que es posible revertir gradualmente los males sociales que trae consigo los excesos de la economía liberal, a partir de la adquisición de competencias cognitivas, afectivas y sociales en los egresados de las instituciones educativas.

Los cambios sociales que tanto deseamos, no se producirán espontáneamente, estos deben iniciarse a partir de quienes compartimos tareas educativas y nos dedicamos cotidianamente a tareas académicas e intelectuales, de ahí la importancia que hayamos iniciado este propósito a partir de la discusión y difusión de la presente lectura, en ambos casos el resultado es beneficioso, todo cuestionamiento o crítica permitirá mejorarla y toda adhesión significará habernos encontrado en un camino que transitamos a diario.

Con esta finalidad los miembros de la Academia Peruana de Psicología hemos construido una biblioteca virtual, con acceso de libre disponibilidad y donde está permitida la reproducción total o parcial de textos, revistas, informes sobre investigaciones y artículos diversos de divulgación científica, cuya dirección: www.academiaperuanadepsicologia.net espera su grata visita. 


\section{Referencias Bibliográficas}

Anojín, P. (1987) Psicología y Filosofía de la ciencia. México: Trillas.

Ausubel, D. (1976) Psicología Educativa: Un punto de vista cognitivo. México: Trillas.

Beigbeder, F. (2002). 13.99 Euros. Barcelona: Anagrama.

Encinas, J. (1934) Ensayo de escuela nueva en el Perú. Lima: Minerva.

Galperin, P. (1976) Introducción a la Psicología. Bs. As: Pablo del Río.

Martel, H. y Urbano V. (2011) Psicología de la actividad psíquica. Lima: UPCUS.

Martel, H. (2012) Excesos de la metodolatría en la investigación científica. Athenea revista virtual de la Academia Peruana de Psicología.

---. (2015) Miseria del positivismo. Horizonte de la Ciencia 5 (9) 95-102.

Maturana, H. (1996) De la Biología a la Psicología. Santiago: Cátedra.

Mosterín, J. (2006) Crisis de los paradigmas en el siglo XXI. Lima: FEUPIGV.

Popper, K. (1974) Conocimiento Objetivo. Un enfoque evolucionista. Madrid: Tecnos.

Rand, A. (2008) ¿Filosofía, quién la necesita? Bs. As: Grito Sagrado.

Sandoval, C. (1996) Investigación cualitativa. Bogotá: ARFO.

Savater, F. (2005). Preguntas de la Vida. Barcelona: Ariel.

Stake, R. (1999) Investigación con estudio de casos. Madrid: Morata.

Tobón, S. (2010) Secuencias didácticas: Aprendizaje y evaluación por competencias. México: Prentice Hall. 